\section{Genetic algorithm assisted error probability optimisation for beamforming}

A. Wolfgang, N.N. Ahmad, S. Chen and L. Hanzo

\begin{abstract}
A novel genetic algorithm (GA) assisted direct error probability optimisation technique is proposed for adaptive beamforming, which reduces the achievable error probability by nearly two orders of magnitude at a signal-to-noise ratio of $10 \mathrm{~dB}$ in the investigated scenario in comparison to the minimum mean-squared error beamforming benchmarker.
\end{abstract}

Introduction: The capacity of wireless systems can be significantly enhanced by separating users transmitting on the same carrier frequency in the spatial domain [1]. Therefore the receiver employs an adaptive antenna array and controls the radiation pattern by adjusting the array weights so that a certain optimisation criterion is met. The conventional beamformer combines the signals received with the aid of each antenna element for the sake of minimising the mean square error (MSE) between a transmitted and a received reference sequence [1] rather than minimising the bit error rate (BER). By contrast, this Letter aims for directly minimising the BER at the beamformer's output, rather than the MSE. A substantial BER performance gain may be achieved at the expense of solving a more elaborate cost function optimisation problem than the minimisation of the MSE. For solving the complex optimisation problem of minimising the BER directly, gradient based algorithms can be employed, although the choice of the appropriate algorithmic parameters may turn out to be challenging. To circumvent these implementational challenges, we propose to employ a random heuristic optimisation algorithm, namely a genetic algorithm (GA) [2] for the direct minimisation of the BER at the beamformer's output.

System model: The desired user and the interferers are assumed to be binary phase shift keying (BPSK) modulated point-sources in the farfield of the receiver. Additionally, we consider a one-dimensional $L$-element antenna array having an inter-element spacing of $d=\lambda / 2$, where $\lambda$ is the wavelength of the sources. The receiver noise $\mathbf{n}$ is assumed to be a complex additive white Gaussian noise (AWGN) process with variance $2 \sigma^{2}$. The antenna array output signal $\mathbf{x}$ at an instant $k$ can then be written as $\mathbf{x}(k)=\mathbf{P b}(k)+\mathbf{n}(k)=\overline{\mathbf{x}}(k)+\mathbf{n}(k)$, where $\mathbf{b}$ is an $M$-element vector containing the symbols of the $M$ number of BPSK modulated users, $\mathbf{P}$ is the $(L \times M)$-dimensional system matrix, with $L$ being the number of array elements and $\overline{\mathbf{x}}(k)$ is the antenna array output signal in the absence of noise. The element $(l, m)$ of the system matrix can be written as $\mathbf{P}(l, m)=A_{m} e^{j \omega t_{l}}\left(\theta_{m}\right)$, where $A_{m}$ is the amplitude of the signal received from the $m$ th user, $\theta_{m}$ is the $m$ th user's angle of incidence and $t_{l}\left(\theta_{m}\right)$ is the relative time delay of the signal transmitted by the $m$ th source, $m=1, \ldots M$, at the $l$ th array element, $l=1, \ldots L$. The beamformer's output can now be expressed as

$$
y(k)=\mathbf{w}^{H} \mathbf{x}(k)
$$

where ${ }^{H}$ denotes the Hermitian operator.

True and estimated BER: It can be shown, that the bit error probability $P_{e}$ encountered at the beamformer's output can be expressed as [3]

$$
\begin{aligned}
& P_{e}(\mathbf{w})=\frac{1}{N_{b}} \sum_{q=1}^{N_{b}} Q\left(g_{q}(\mathbf{w})\right) \quad \text { with } \\
& g_{q}(\mathbf{w})=\frac{\operatorname{sgn}\left(b_{q, 1}\right) \operatorname{Re}\left\{\mathbf{w}^{H} \overline{\mathbf{x}}_{q}\right\}}{\sigma_{n} \sqrt{\mathbf{w}^{H} \mathbf{w}}}
\end{aligned}
$$

where $N_{b}=2^{M}$ is the number of possible transmitted bit sequence combinations of the $M$ BPSK users, $\mathbf{b}_{q}$ is the $q$ th possible transmitted bit sequence with $1 \leq q \leq N_{b}$ and $\overline{\mathbf{x}}_{q}=\mathbf{P} \mathbf{b}_{q}$. The symbol $b_{q, 1}$ is assumed to correspond to the desired user's BPSK symbols. To aim for a realistic receiver structure, the true bit error probability $P_{e}$ is replaced by its estimated value $\hat{P}_{e}$, which can be obtained using kernel density estimation [4]. The estimated bit error probability $\hat{P}_{e}$ may be written as [3]

$$
\begin{aligned}
& \hat{P}_{e}(\mathbf{w})=\frac{1}{K} \sum_{k=1}^{K} Q\left(\hat{g}_{k}(\mathbf{w})\right) \quad \text { with } \\
& \hat{g}_{q}(\mathbf{w})=\frac{\operatorname{sgn}\left(b_{1}(k)\right) y_{R}(k)}{\rho_{n} \sqrt{\mathbf{w}^{H} \mathbf{w}}}
\end{aligned}
$$

where $K$ is the reference sequence. length, $y_{R}(k)$ is the real part of the received reference symbol, $b_{1}(k)$ is the transmitted reference symbol and $\rho_{n}$ is the so-called kernel width, also known as the smoothening parameter. Note that for the kernel density estimation of the probability density function (pdf) of $y_{R}$ a Gaussian kernel function was used, which has been transformed into the $Q$-function by integrating it. The challenging and novel task of the GA in the system configuration considered is to calculate the complex array weight vector $w$ in order to minimise (3).

BER surface: In Fig. 1 the BER surface described by (2) is shown for a two-element array detecting five equal-power users arranged according to the angular positions of Table 1 . The imaginary part of the beamformer weights was fixed to the optimum solution. The minimum of the BER surface shown can only be found with the aid of a gradient based algorithm if it is initialised appropriately, since the surface is characterised by a narrow valley representing the lowest achievable BER solution. If the error surface is estimated using (2) it will additionally become irregular and thus gradient based algorithms may not converge to the minimum BER solution. More explicitly, for sub-optimum step-size settings, the gradient based algorithms may get trapped in a local minimum of the BER surface described by (2) and (3), thus resulting in a sub-optimum solution [3]. The motivation of our research was to derive a GA, which is capable of finding the antenna array weights that directly minimise the BER at the beamformer's output and therefore circumvents the problems imposed by gradient based algorithms.

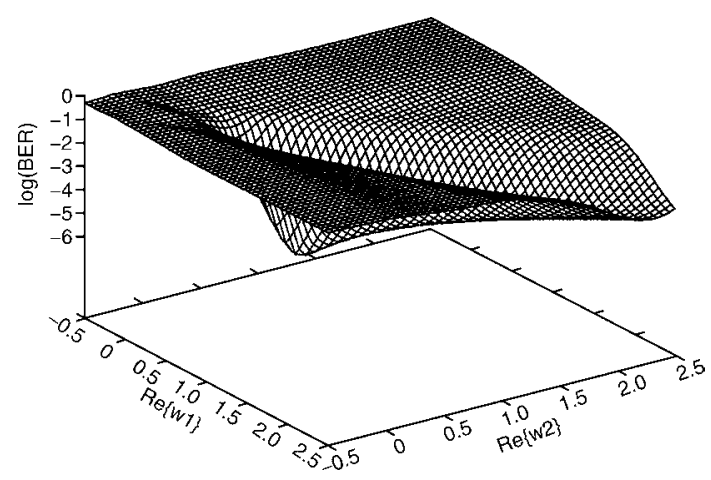

Fig. 1 BER surface for two-element linear array and five equal-power sources at $S N R=10 \mathrm{~dB}$ arranged according to Table 1 Imaginary part of weights fixed to minimum BER solution

Table 1: Angles of incidence relative to perpendicular of antenna array

\begin{tabular}{|c|c|}
\hline User & Interferer \\
\hline$\theta_{0}=15^{\circ}$ & $\theta_{1,2,3,4}=-30^{\circ}, 60^{\circ},-70^{\circ}, 80^{\circ}$ \\
\hline
\end{tabular}

Genetic algorithm: The genetic approach can be interpreted as a guided random search process, which attempts to imitate biological evolution [2]. The GA commences its iterations with a set of potential array weight solutions referred to as the initial population, which can be chosen randomly from within a given search space. For each of these potential initial solutions, which are also referred to as GA individuals, the so-called fitness function is evaluated, as follows

$$
f_{i}=1-\frac{1}{1-\log \left(P_{e}\right)}
$$

which approaches unity as $P_{e}$ decreases. This function describes the quality or fitness of a potential solution and ensures that the most fit GA 
individuals are selected as 'mates' of the GA creating 'offspring', i.e. new array weight estimates, which become part of the next generation. By successively repeating the procedure of combining the best array weight estimates of the previous generation for the sake of creating new estimates, the algorithm tends to converge to the best solution for the fitness function, namely to the minimum BER solution. The parameters determining the complexity of the GA are the number of individuals in a population (population size $P$ ) and the number of iterations the GA has to invoke (number of generations $G$ ). These two determine how often the fitness function has to be evaluated. The GA operators [2] used in our GA setup, namely, roulette selection, single point crossover, bit-inversion as mutation type, elitism, span scaling, incest prevention and weighted mutation are here only mentioned for the sake of completeness.

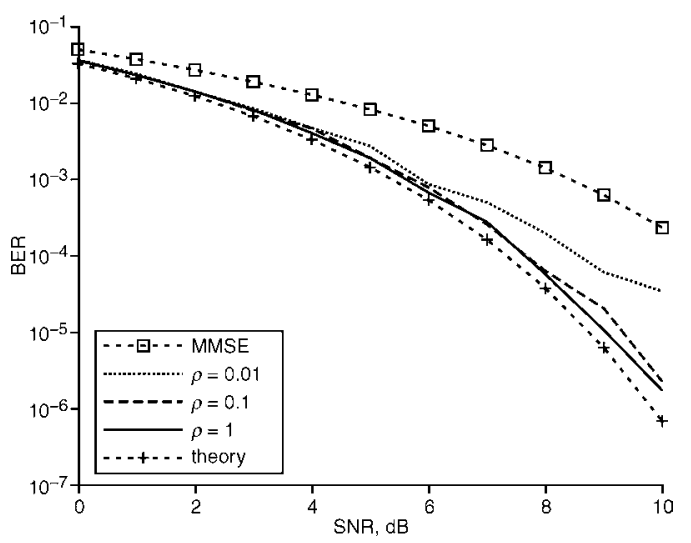

Fig. 2 BER against SNR for two-element linear and five equal-power sources arranged according to Table 1

GA employed had population size of $P=40$ and $G=40$ number of generations. The BER associated with specific values of fitness function of (4) estimated using (3) for different values of $\rho$

Simulation results: For the sake of arriving at the analytical minimum BER expression of (2), a conjugate gradient algorithm based array weight adaption procedure has been proposed in [3]. This theoretical minimum BER performance bound will serve as our first benchmarker, when the performance of the GA is evaluated. The second bench-marker is the MMSE solution given as [3] $\mathbf{w}=\left(\mathbf{P P}^{H}+\right.$ $\left.2 \sigma^{2} \mathbf{I}_{L}>\right)^{-1} p_{1}$, with $\mathbf{I}_{L}$ being the $(L \times L)$-dimensional identity matrix and $p_{1}$ being the first column of $\mathbf{P}$. For a given weight vector calculated, the BER was evaluated using (2), so that resorting to Monte Carlo simulation could be avoided. The BER curves presented were averaged over $1000 \mathrm{GA}$-aided weight-optimisation runs. For our investigations we used a two-element linear array with an interelement spacing of $\lambda / 2$. The users were arranged according to Table 1. It can be seen from Fig. 2 that for a reference sequence length of $K=256$ and for $P=40$ as well as $G=40$ the algorithm is capable of converging for a wide range of kernel width values $\rho$. As expected, for an inappropriately chosen value of $\rho_{n}$ the minimum of the BER surface is shifted away from the theoretical solution. In this case the GA becomes incapable of converging to the minimum BER solution. In the first study we used a fixed kernel width of $\rho_{n}$, but ideally the kernel width has to be adjusted according to the variance of the received training sequence [4] and is thus dependent on both the SNR and the INR. Silverman has provided a simple rule of thumb for the estimation of $\rho_{n}$ given as [4]

$$
\rho_{n}=\left(\frac{\hat{\sigma}^{5}}{3 K}\right)^{1 / 5} \simeq 1.06 \hat{\sigma} K^{-1 / 5}
$$

where $K$ is the reference sequence length and $\hat{\sigma}$ is the standard deviation of the received reference sequence. Equation (5) tends to over-smoothen the BER estimate, but the results presented in Fig. 2 suggest that the GA is capable of compensating for this inaccuracy. Fig. 3 shows the achievable BER against SNR performance for $P=50$ and $G=30$, when detecting equal-power users, as well as for a scenario studying the near-far effect, where the signals of the interferers at $\theta_{1,2}=-30^{\circ}, 60^{\circ}$ were received with $6 \mathrm{~dB}$ higher power than the desired user's signal. The graphs illustrate the superiority of the minimum BER approach over the MMSE approach in terms of counteracting the near-far effects, while demonstrating that the GA is capable of automatically adapting to the new BER surface without being reconfigured, although a shorter reference sequence of $K=64$ is now used instead of $K=256$.

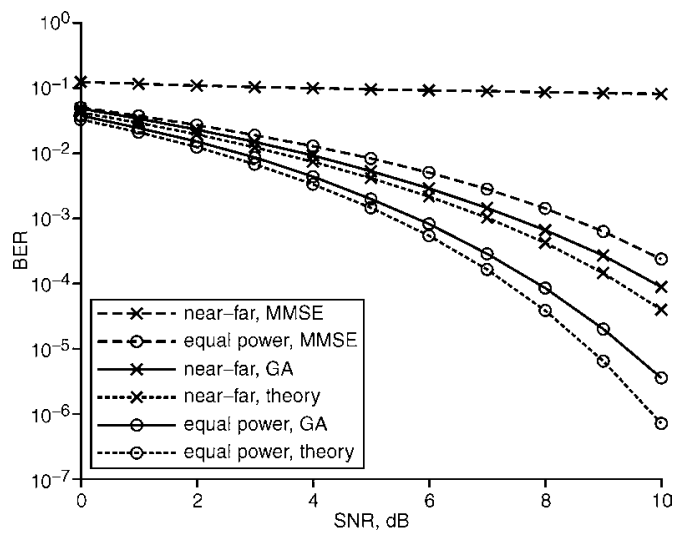

Fig. 3 BER against SNR for five equal-power sources and for near-far constellation, where the two interferers having angles of incidence $\theta=$ $-30^{\circ}$ and $\theta=60^{\circ}$ had $6 \mathrm{~dB}$ higher power than desired source

GA employed had population size of $P=50$ and $G=30$ number of generations. The BER associated with specific values of fitness function of (4) estimated using (3) and (5)

(C) IEE 2004

3 December 2003

Electronics Letters online no: 20040229

doi: 10.1049/el:20040229

A. Wolfgang, N.N. Ahmad, S. Chen and L. Hanzo (School of ECS, University of Southampton, Southampton, SO17 1BJ, United Kingdom)

\section{References}

1 Blogh, J.S., and Hanzo, L.: 'Third-generation systems and intelligent wireless networking - smart antennas and adaptive modulation' (John Wiley \& IEEE Press, 2002)

2 Hanzo, L., Yang, L.-L., Kuan, E.L., and Yen, K.: 'Single- and multicarrier DS-CDMA' (John Wiley \& IEEE Press, 2003)

3 Chen, S., Hanzo, L., and Ahmad, N.N.: 'Adaptive minimum bit-error rate beamforming assisted receiver for wireless communications'. Proc. ICASSP 2003, 2003, Vol. 4, pp. 640-643

4 Silverman, B.W.: 'Density estimation' (Chapman Hall, London, 1996) 